\title{
CDISC SDTM ARC Coronary Stent Thrombosis Grade Responses Terminology
}

National Cancer Institute

\section{Source}

National Cancer Institute. CDISC SDTM ARC Coronary Stent Thrombosis Grade Responses Terminology. NCI Thesaurus. Code C119020.

Terminology associated with the ARC coronary stent thrombosis grade responses codelist of the Clinical Data Interchange Standards Consortium (CDISC) Study Data Tabulation Model (SDT M). 